\title{
Nopartmentof
}

Journal of Rehabilitation Research

and Development Vol. 29 No. 4, 1992

Pages 64-77

\section{A human factors approach to adapted access device prescription and customization}

\author{
Sharon August, MSc, OT(C) and Patrice L. Weiss, PhD, OT(C) \\ School of Physical and Occupational Therapy, McGill University, Montreal, Quebec H3G 1 Y5 Canada
}

\begin{abstract}
Adapted access device prescription and customization is often a lengthy and cumbersome process. To date, few objective procedures are available to assist in the prescription process. Rather, clinician and client rely on a trial-and-error approach that is often severely constrained by the size of their adaptive device collection as well as the extent of clinical expertise. Furthermore, the large number of available options and lack of information delineating the mechanical and physical characteristics of these devices means that therapists must take time away from direct clinical contact to probe each adaptation in detail. There is available in the human factors domain a body of literature that is highly relevant to adapted access. Of particular interest are the studies that have addressed issues related to the suitability of standard and alternative input devices in terms of task productivity (via improvements in input speed, accuracy, and endurance), and their ability to minimize the risk of acute and chronic work-related dysfunction. This paper aims to consider the relevance of human factors research for physically disabled individuals. Three human factors issues-digit travel, digit loading, and device positioning-have been selected as representative of factors important in the configuration of adapted access devices.
\end{abstract}

Key words: adapted access, human factors, keyboard, microcomputer, physical disability.

Address all correspondence and requests for reprints to: Dr. P.L. Weiss, School of Occupational Therapy, Hebrew University, Mount Scopus, P.O. Box 24026, Jerusalem 91240 Israel.

S. August is now affiliated with the Jewish Rehabilitation Hospital, Laval, Quebec, and Dr. Weiss is now affiliated with the School of Occupational Therapy, Hebrew University, Jerusalem, Israel.

\section{INTRODUCTION}

Access to microcomputers for the physically disabled

One of the major applications of computer usage in rehabilitation has been the use of microprocessor-based adaptations to expand the opportunities of people who have limited physical abilities. Those lacking sensory-motor and/or cognitive-perceptual skills have achieved greater independence when carrying out vocational and avocational tasks with microcomputer assistance.

Because of the legibility of the finished product and the flexibility of operation, keyboard access has been one of the most popular methods of written communication by the physically disabled (1). Within the last decade, numerous adaptations have been developed to enable computer access. The potential benefit of these adaptations is so great and the number of options so extensive that in recent years, a subspecialty known as adapted access has evolved. Essentially, this field entails the prescription and customization of hardware and software to facilitate such tasks as communication and other activities of daily living. Device prescription involves the replacement of those functions normally accomplished by standard input devices (e.g., keyboards and pointers) with alternative devices that are capable of modifying, substituting, or emulating these standard data entry modes. Device customization entails modification of the device to suit the specific abilities and needs of the client best $(2,3)$. 
To achieve this goal, clinicians typically use a hierarchical approach that is based on prescribing the device with the greatest potential for productivity. In the simplest approach, minor modifications to standard computer hardware and/or software are prescribed. Aimed at those individuals whose physical abilities permit them to enter information in a direct and efficient manner, these adaptations modify the standard input methods rather than replace them. For example, simultaneous multiple key presses can be provided, when needed, by programmable keylocks or can be prevented, when an impediment, by keyguards.

At the next level, alternative keyboards or pointers substitute for standard input devices. These are coupled to microcomputers via special firmware cards or RAM-resident programs. Each of these alternatives has been designed to accommodate a particular type of access problem by enhancing the residual ability of the individual and minimizing the need for skills that are weak or lacking. For example, a quadriplegic client who must rely on a mouthstick may benefit from the prescription of a programmable minikeyboard that requires less keyto-key movement.

Data entry emulation is often the only intervention suitable for severely physically disabled individuals. In such cases, physical ability is so limited that the individual is unable to access microprocessorbased devices except by an indirect method. This approach involves the activation of one or more switches by a body part most able to execute precise and controlled actions, such as raising an eyebrow or puffing on a tube. At this level, scanning or encoding are the two most common options. Scanning entails the use of successive switch activations to select letters from a matrix of characters displayed on a monitor. Morse code is executed by the activation of different combinations of one or two switches that encode the entire alphanumeric set. Further details on adapted access methods, as well as examples of specific adaptations, are widely available in the literature $(1,2,3,4,5)$.

\section{Limitations of current adapted access practice}

Regrettably, adapted access device prescription and customization is often a lengthy and cumbersome process. To date, few objective procedures are available to assist in the prescription process. Clinician and client are forced instead to rely on a trial-and-error approach that is often severely constrained by the size of the adaptive device collection as well as the extent of clinical expertise. Furthermore, the large number of available options and lack of information delineating the mechanical and physical characteristics of these devices mean that therapists must take time away from direct clinical contact to probe each adaptation in detail (6). As the number and types of devices continue to increase, the already arduous selection process will become even more formidable.

\section{Enhancing the current practice of adapted access}

Although few clinical studies have explicitly examined the properties of adapted access devices $(6,7)$ and characteristics of device usage, there is available in the human factors domain a body of literature that appears to be highly relevant to adapted access. Human factors is defined as the systematic application of information about human abilities and behavior to the design and evaluation of equipment and the environment in which it is used (8). It includes a set of experimental and analytic tools for the quantitative assessment of how well any given object can be used by an individual having specific capabilities. Research in this field has been pivotal in focusing attention on improvements in workspace design and human-machine interfaces (8). Of particular interest to the area of adapted access are the studies that have addressed issues related to the suitability of standard and alternative input devices in terms of task productivity (via improvements in input speed, accuracy, and endurance) and their ability to minimize the risk of acute and chronic work-related dysfunction.

The human factors domain represents a rich source of potential data. These data, however, must be applied with caution to adapted access device prescription. Given the often severely limited physical abilities of the users of this equipment (e.g., decreased speed and endurance), one may not assume the applicability of data based on experimental studies of subjects whose functions constitute the norm.

\section{METHOD}

This paper aims to consider the relevance of human factors research for this clinical population. 
Journal of Rehabilitation Research and Development Vol. 29 No. 4 Fall 1992

Three specific issues (digit travel, digit loading, and device positioning) have been selected to illustrate the diversity of available material and to show how human factors data may or may not be of relevance in the adapted access prescription process. As detailed in the following sections, these particular issues were chosen because: 1) they address some of the major difficulties experienced by disabled users, and 2) they embrace a variety of ergonomic alternatives ranging from those commonly prescribed to those rarely considered. It is anticipated that this material will serve as a foundation to help clinicians substantiate the prescription of currently used adapted access options to those in positions of administrative or financial authority. It is further anticipated that this material will increase clinicians' awareness of data entry options that have, to date, received too little consideration.

\section{Digit travel}

Digit travel can be defined as the displacement traversed by the digits in the horizontal or vertical planes as successive keyboard keys are activated. As a result of the mechanical limitations of the early machines, digit travel in both planes was exceedingly large. The spring-lever typewriting mechanism involved the depression of a metal key by more than $150 \mathrm{~mm}$ which, in turn, rotated an engraved metal rod to impress a character on paper. Adjacent rods would obstruct each other when rotated in too quick succession $(9,10)$. The need to accommodate these mechanical components and to permit their satisfactory functioning apparently led to the fabrication of large, sharply inclined keyboards and to the adoption of the familiar QWERTY alphanumeric layout.

The advent of electromechanical typewriters and, more recently, electronic keyboards, has removed many of the constraints formerly imposed by their mechanical counterparts. Key depression displacement has been reduced and the magnitude of the keyboard profile and footprint are no longer confined by the size of the internal components. This flexibility combined with the wish to increase keystroke rate and decrease data entry fatigue has engendered a proliferation of novel keyboard designs. Many of these design recommendations have been directed at reducing digit travel for both experienced and novice typists $(9,11)$. Included in these changes are the reorganization of the alphanumeric layout, major hardware innovations such as miniature and chord keyboards, novel methods of data entry, and the modification of the displacement properties of individual keys.

Alphanumeric Layout. It is probable that the standard QWERTY layout resulted from the need to limit typing speed by compelling the digits to traverse relatively large distances as successive letters were typed $(12,13,14)$. Many yardsticks have been used to quantify the extent of digit travel and its implications for efficient keyboard usage. One of the most common approaches involved frequency counts of the location of the letters comprising commonly occurring character strings, words, and phrases. For example, Ferguson and Duncan (15) recommended that a more efficient layout would avoid the placement of such letters (e.g., ing, ere) on keys located in the front and back rows; this is a common occurrence with the QWERTY design. One study showed that 52 percent of QWERTY typing is done on the back row, 16 percent on the front row and 32 percent of the words on the home row (9).

A major objective of Dvorak's (11) novel alphanumeric layout was to diminish digit travel. This keyboard is considered by some to be an optimal layout because it permits the typing of an exceptionally large number of commonly used words exclusively with home row characters (12). Despite the considerable interest in this layout $(9,11,16)$ the Dvorak keyboard has not been widely adopted. Although some studies have shown it to be easier to learn and to result in greater speed and accuracy $(11,17)$ others dispute these findings $(14,16)$ and suggest that improvements of less than 10 percent are more realistic $(8,18)$. Unfortunately, most comparisons of the QWERTY and Dvorak keyboards have been confounded by the familiarity of the subjects with only one of the layouts. To date, the literature has not provided strong enough support for the Dvorak's advantage to warrant its replacement of the QWERTY.

It is interesting to note that Noyes (10) argued that the QWERTY keyboard may be well planned precisely because it does require that the typist use numerous finger movements. The kinesthetic cues that arise from such movement may provide valuable feedback to the typist (19). It appears that the reliance on kinesthetic feedback rises rapidly from low levels among inexperienced typists and plateaus for intermediate and expert typists because the latter rely more on this sensation for feedback (19). 
Alternation of Hands and Digits. The hand and finger movements of novice and proficient keyboard operators are noticeably different. Skilled typists keep their hands in almost continuous motion with a fluid overlapping of movement between hands and among the digits (20). Conversely, unskilled typists rely on a slow and awkward hunt-and-peck method using one or more fingers. With the latter typing technique, each keystroke must be made in sequence leaving no opportunity to overlap keystroke movements and diminish the interkeystroke interval $(20,21)$. This observation has led to the suggestion that the smooth interaction among digit movements may be a key factor in achieving faster and more efficient typing performance $(13,20)$.

A number of studies have demonstrated that the interkeystroke interval, duration of key contact, and magnitude of key activation force diminished when pairs of letters (digrams) were typed by alternating digits on each hand than when different digits on the same hand were used $(13,14,22,23)$. One of the major criticisms of the QWERTY keyboard layout related to the fact that it does not make sufficient use of alternating hands (14) and that a disproportionate number of words are typed solely by the left hand $(11,14,15)$. In contrast, the Dvorak layout is thought to promote digit alternation (14).

Miniature Keyboards. Reduction of key and keyboard size is another way to minimize digit travel. Public demand in recent years has encouraged the major manufacturers of electronic equipment to produce smaller and lighter credit card calculators, electronic agendas, and notebook computers. This interest in miniaturization has been motivated primarily by a growing appreciation of the convenience and efficiency of portable units. Regrettably, such factors have had a much greater impact on the design and construction of these devices than have considerations of user accuracy, speed, and productivity. In view of the very large differences between the physical dimensions of standard (approximately $190 \mathrm{~mm}^{2}$ ) and miniature (approximately $30 \mathrm{~mm}^{2}$ ) keys, it is highly likely that significant differences in performance do occur. However, user performance on miniature keyboards has not been extensively examined. The only available systematic study examining the effect of key size on ten-key push button telephone sets was published more than 30 years ago (24). It is doubtful that these data are relevant to performance on modern electronic keyboards.

Chord Keyboards are devices that encode the alphanumeric set via the coordinated activation of one or more keys. Digit travel in the horizontal plane is reduced because these keyboards require fewer than a tenth of the keys used in standard configurations. This economy of movement is achieved, however, at the expense of increased mental effort (primarily directed at learning the chords) and the fine motor skill of coordinating the simultaneous activation of keys (10).

Typing performance with both unilateral and bilateral chord keyboards has been examined $(25,26)$. Perhaps as an outcome of methodological factors such as the manner of text presentation, differences in spatial orientation of the keyboards, and the use of a foreign language in one of the studies, the results of these two investigations were contradictory. Whereas Ratz and Ritchie (26) demonstrated that one-handed chord keyboard typists were faster than those who typed with the twohanded version, Gopher and Raji (25) showed the reverse. Both unilateral and bilateral chord keyboards seemed to enable typists to achieve typing speeds approximately 10 percent faster than possible with the standard QWERTY layout $(25,26,27)$. It is generally accepted that individuals with at least moderate cognitive abilities and sufficient isolated digit control do not require extensive training to master chord keyboards $(25,27,28)$. For example, Gopher and Raji's subjects were able to memorize chord key sequences in less than 1 hour and achieved rates of about 40 words per minute after 35 hours of training (25). Nevertheless, the potential benefits of chord keyboards do not seem to outweigh the efforts that would be required to replace the QWERTY (10).

Data Entry Method. Traditionally, the keyboard was viewed as the primary method of data entry because it resulted in greater speed and accuracy for alphanumeric input (29) when compared with control devices such as wheels, knobs, and levers (8). With the recent surge of window environment software, menu-driven interfaces, and graphic programs, cursor-control pointing devices have become an increasingly important method of data entry. This is significant because alternate modes of data entry, such as the mouse, trackball, and touch pad, have the potential to reduce activa- 
Joumal of Rehabilitation Research and Development Vol. 29 No. 4 Fall 1992

tion force requirements and relieve the digits of a substantial part of their workload. The most popular cursor-control devices, including the mouse, joystick, and trackball, can be used to operate all pointing-based software. Other devices, such as the touch screen, touch pad, light pen, and digitizing tablet, appear to be suited to specific tasks. In a study of pointing accuracy and speed, Albert (30) compared the standard keyboard with five of the major cursor-control methods. The trackball was considerably more accurate than all other devices, whereas the touch screen was the least accurate. In contrast, the touch screen was the fastest control method, whereas the keyboard was the slowest. The selection of the best data entry device must take into account both the nature of the task (alphanumeric versus cursor control) and the relative importance of speed and accuracy.

Key Displacement. Vertical digit travel, which occurs as each key is depressed, can be reduced considerably by the use of different key activation mechanisms. The typical plastic key-cap perched atop a spring requires activation displacements ranging from 2.5 to $16 \mathrm{~mm}(6,9,31,32)$. In contrast, membrane keyboards and switches require virtually no displacement $(7,32)$. It is interesting to note that touch typists at all skill levels were faster when they used the more familiar conventional keyboard (20 to 43 words per minute) than when they used the membrane keyboard ( 20 to 32 words per minute) although these differences decreased as the subjects had further opportunity to use the two keyboards (32). No significant differences in accuracy were observed.

Clinical Relevance. Vertical digit travel, altered primarily via changes in key activation mechanisms, has decreased dramatically with the advent of electronic keyboards. In contrast, horizontal plane digit travel, which can be reduced significantly via changes in keyboard appearance (alphanumeric layout, miniature keyboards) and access method (alternation of hands and digits, chording), has not had a significant impact on standard keyboard usage. For most users, the relative advantage to be obtained from these innovations does not seem sufficient to offset the cost of teaching new typing techniques or replacing equipment.

The cost-benefit trade-off with respect to the disabled population is very different. Physically disabled individuals who lack the redundancy of abilities to compensate for poorly designed equipment are much more in need of these ergonomic alternatives. Their involuntary reliance on such slow and inefficient data entry techniques as single-digit typing or stick access (by means of the head, mouth, or universal cuff) necessitates the exploration of innovative access techniques. In such cases, the time required to master a chord-based access method, the cost of acquiring a keyboard that permits modification of the alphanumeric layout and the psychological readiness to embrace these changes are more acceptable to the user. Moreover, the relatively small improvements of speed, accuracy, and endurance secured by the adoption of these alternatives may be more meaningful within the context of the performance levels of the user with a disability. In the most severe instances such modifications may be the only choice for successful access.

A number of access methods that focus on the minimization of digit travel have recently been applied to disabled computer users. Chubon and Hester (1) showed that single-digit typing was improved with a center-weighted keyboard that was reconfigured according to letter frequency data. This keyboard is a more appropriate alphanumeric layout for single-digit typing, because, in contrast to the multifinger Dvorak layout, travel is minimized with respect to the center of the keyboard. Kirschenbaum et al. showed that typing on a unilateral chord keyboard required less effort by children with spastic and athetoid cerebral palsy and progressive muscular dystrophy (28).

Not all of the ergonomic solutions that reduce digit travel are applicable to users with disabilities. For example, the manipulation of the alphanumeric layout to promote alternation of hands has been shown experimentally to be a factor that improves rates of data entry by approximately $30-50 \mathrm{~ms}$ per digram $(13,21,33)$. Given the access speeds of even the fastest single-digit typists, the relevance of this ergonomic solution is doubtful.

\section{Digit loading}

Digit travel is not the only human factors criterion that has profited from the greater flexibility of modern keyboard technology and from the development of innovative software algorithms. Digit loading, defined as the force that must be exerted by the digits to activate the keyboard keys, is now substantially more accessible to modification 
than in the past. This is important because the structure and function of most standard keyboards was not well matched to the relative capabilities of the digits. The QWERTY keyboard, originally designed to be used with a hunt-and-peck mode of typing, does not even consider individual digit loading (11). Typical key surfaces were similarly sculptured, their activation properties were identical, and they had been arranged with little recognition of either absolute or relative digit strength $(9,34)$, dexterity (11), and susceptibility to fatigue (9). Only the thumb, by virtue of its distinctive anatomical position, was allocated a unique function in keyboard usage (12). Ironically, despite the fact that it possesses the greatest strength and degrees of freedom, the thumb is generally allocated the least amount of work $(11,15)$.

Excessive loading of the digits over extended periods of time is recognized as a source of acute and chronic occupational distress $(9,15)$. It is also one of the factors limiting computer usage by the physically disabled. The magnitude and distribution of activation force of standard keyboards are usually beyond the capabilities recommended for those who are suffering from severe degenerative neuromuscular pathology. In addition, the undesirable deformities associated with other chronic conditions, including rheumatoid arthritis and chronic peripheral neuropathies, are exacerbated by standard keyboard usage patterns. For example, keys that require high activation force may push a digit into hyperextension and place undue stress on joints that are suffering from ligament laxity.

Digit loading can be effectively altered via a number of ergonomic alternatives. The use of switch mechanisms of varying sizes and components can modify the magnitude of the activation force. Alternate modes of data entry such as the mouse, trackball, and touch pad described above have the potential to reduce activation force requirements and relieve the digits of a significant part of their workload. Finally, software designed to implement macros or word prediction algorithms improve data input/output efficiency to minimize unnecessary keystrokes.

Switch Mechanism. In direct contradiction to human factors design principles, the magnitude of the force required to activate the keys on the early mechanical keyboards, on electric typewriters, and on most modern computer keyboards was not based on studies of human operator abilities. Rather, de facto standards evolved as a function of the prevailing switch technology (e.g., spring versus contact mechanisms) and have persisted as a result of convention (9). Only a few studies have identified activation force data for manual typewriters (9), electric typewriters, conventional $(7,32)$ and membrane computer (32) keyboards and special keyboards adapted for people with disabilities (7). These data are of limited value, however, because: 1) their reliability and validity have not been well established; 2) they identify only the static mechanical characteristics (8); and, 3) they do not take into account changes resulting from prolonged and repeated usage.

Membrane-like switches, used with increasing frequency to operate appliances, entertainment units, and electronic keyboards, represent the most significant attempt to make use of changes in switch mechanism. Contrary to the typical user's assumption (7), the activation of these switches is not associated with large reductions in digit loading (although digit travel is greatly reduced). In fact, membrane switches require more force to operate than do conventional push buttons (200-500 g versus $50-90 \mathrm{~g}$ ), apparently to help reduce accidental activation $(7,8,32)$. Moreover, the absence of both auditory and tactile keystroke feedback has been shown to have a detrimental effect on speed, accuracy, and user preference (31).

Clinical Relevance. The use of different switch mechanisms, data entry methods, and algorithms that improve input/output efficiency all have considerable potential for facilitating computer usage by the disabled. Digit loading may be altered by the replacement of the standard key-cap springs (35) or by the redistribution of relative load with the use of the center-weighted layout (1). An alternative data entry device, such as the trackball, may be recommended for a client with rheumatoid arthritis rather than a joystick, which tends to require excessive grip force. Finally, algorithms such as macros, more commonly known in clinical settings as abbreviation-expansion techniques, are frequently used.

Communication systems for nonvocal individuals who are physically disabled, and other programs, have been developed to enable individuals to enter a code of minimal characters for a word, thereby leaving the computer to complete the word $(1,36)$. The need to remember various codes may be 
Journal of Rehabilitation Research and Development Vol. 29 No. 4 Fall 1992

demanding for some individuals. Word prediction is an alternative method to enhance input/output ratio. The technique was developed in an attempt to eliminate the memorization of codes necessary for abbreviation-expansion (36). Rather than having to rely on abbreviated commands and phrases, the computer is programmed to predict plausible completions to the initial characters entered into the computer by the operator. For example, subsequent to entering initial characters such as "ex" a list of words such as exaggerate, except, explore, or extra is presented. The appropriate word can then be selected by a single keystroke, thereby reducing the total number of entries $(4,36,37)$. The possibility of complete sentence generation is also being investigated (36).

\section{Device positioning}

The application of ergonomic principles to the positioning of users with disabilities and to the placement of the devices they use is a fundamental component of the adapted access prescription process at all levels of intervention. Thus, issues related to device height, proximity, and angle are applied to the placement of a keyguard, alternative keyboard, or switch. Therapists readily manipulate the position of a device because such variations are postulated to have a considerable influence on the upper extremity postures required for device operation. These postures, in turn, are thought to influence data entry productivity, user comfort, and the development of both short-term and chronic clinical symptoms $(34,38,39)$.

The ergonomic literature provides considerable evidence that poor positioning is the source of biomechanical and physiological problems. Experimental results based on visual observation, videotaping, force transducer measures, and electromyographic recordings have consistently demonstrated that the upper extremity and trunk postures adopted by both experienced and novice typists when using standard equipment have serious biomechanical and physiological consequences $(20,34,38,40)$. For example, in the classic typing position, the typically high placement of the keyboard induced shoulder elevation which, in turn, caused the shoulder girdle musculature to assume a shortened position (40).

Access to the standard keyboard also required that the pronator muscles be maintained for prolonged periods in a shortened position (40). To alleviate the resultant tonic muscle tension, typists tended to assume postures that lead to excessive shoulder abduction, which in turn increased ulnar deviation (38). These postures were found to be correlated with chronic neuromuscular symptoms and it was postulated that the associated soft tissue stress was a cause of common clinical symptoms (15). There appears to be little doubt that long-term, full-time use of standard keyboards without proper placement leads to occupational difficulties $(15,41)$.

One of the most frequently suggested solutions to the above problems deals with changes in keyboard height. Although most investigators agree that keyboard height is one of the most important factors in achieving comfortable and efficient usage during prolonged data entry tasks, there appears to be an extremely wide range in the values recommended for the population at large $(38,42,43)$. This presumably reflects a wide range of user preferences. As a result, most researchers only publish general guidelines (such as, the work surface should be at a level that permits the arms to hang relaxed from the shoulder with the forearm near horizontal or slightly lower) and recommend that provision be made for adjustment of work surface height to suit the physical dimensions and preferences of the user (8).

Keyboard slope is another ergonomic solution that has been widely adopted by keyboard manufacturers in recent years. For a variety of reasons, including user preference $(43,44)$, biomechanics of optimal hand function (45) and reduced resting EMG levels (40), most current keyboard models come with some amount of slope, varying from 10 to 30 degrees (9). Added adjustability is provided by means of mechanical levers. The major exceptions to this are the newer hand-held "notebook" computers, such as the Casio and Sharp, which emphasize a small and thin profile.

Split Keyboards. In view of the importance of keyboard height and slope, it is surprising that ergonomic investigations have not resulted in more definitive conclusions. In contrast, split and laterally tilted keyboards, a relatively rare approach to data entry, have been the subject of fairly intensive study. Over the years, the "split" keyboard, a novel design in which the keyboard is divided into two symmetric half-keyboards, has been periodically suggested to reduce the incidence of chronic neuromuscular symptoms. 
The first split keyboard, constructed by Klockenberg (34), was designed to provide faster, more accurate, and less fatiguing keyboard access. Essentially, the alphanumeric keys were distributed equally on two separate sections each hinged to a horizontal centerpiece (34). Each section could be laterally tilted at angles varying from 30 to 90 degrees. Creamer and Trumbo (46) studied an eight-key, split keyboard that was laterally tilted at angles ranging from 22 to 66 degrees. In this study, lateral tilt appeared to increase key press rate and decrease errors but did not alter the fatigue perceived by users. These findings were contradicted by Kroemer (34), who studied how typing performance varied as a function of the degree of lateral tilt on a remodeled Klockenberg keyboard. Although he did not demonstrate any significant improvements in typing speed or accuracy for either trained or untrained typists, his subjects claimed to feel more comfortable with the split, tilted keyboard. Furthermore, the lateral tilt (when in the range of 10 to 30 degrees) decreased muscle activity in the shoulder girdle and arm region, suggesting more comfortable keying (38).

Nakaseko, et al. (38) added an additional variable, the opening angle, and looked at changes in combinations of these two factors in terms of experienced typist preference as well as body postures (obtained with static goniometric measurements). In comparison to a standard keyboard, the split, open-angled keyboard was found to decrease ulnar deviation from 20 to 10 degrees, which is in the range associated with the least incidence of neuromuscular pathology.

Clinical relevance. The orientation and placement of low- and high-technology assistive devices has long been accepted by therapists to be of great importance to the productivity, comfort, and wellbeing of the client $(2,3)$. Fortunately, the readily available and diverse selection of adjustable workstation furniture provides therapists with easy and flexible ways to achieve a suitable setting for keyboard and monitor height, slope, and proximity. In the absence of widely accepted standards for device placement, ergonomic guidelines gleaned from the human factors literature are used only in a general way. The specific values that will promote optimal device positioning for both pediatric and adult populations remain to be identified. Extrapolating anthropometric standards to children or adults whose stature is extremely limited may be misleading because of pathological differences (e.g., severe scoliosis and kyphotic deformities, arthritis). User preference appears to be very important, although the therapist must be careful to remain within ergonomic guidelines and respect biomechanical principles so that poor habits do not supersede improved function and encourage the development of deformities.

In contrast to device height and slope, a number of positioning options have not attained wide recognition despite the abundant literature that demonstrates their role in promoting user comfort, preventing deformity, and increasing productivity. The use of split and laterally tilted keyboards is one example of an ergonomically derived solution that has had remarkably little influence in the general work force or clinical applications. Presumably, this lack of use is the result of unawareness on the part of those who could benefit from the solution. It is recommended that therapists become more familiar with these novel keyboard alternatives.

\section{RESULTS}

The diversity of adapted access approaches and the profusion of adaptive devices oblige clinicians to select what appear to be the most feasible options from among a small subset of the alternatives known to them. It is often difficult to ascertain whether one of the standard adapted access solutions (e.g., scanning) is suitable to a particular client or whether a less conventional approach (such as a chord keyboard) should be considered. In our own clinical work, we attempt to cope with this difficulty by drawing on results from the human factors literature to 1) support our selection of standard choices and 2) explore novel approaches to adapted access.

Thus, we critically review the literature relating specific human factors issues (digit loading) to ergonomic solutions (input/output efficiency) that can improve task productivity and minimize disability formation. We then evaluate their applicability to a physically disabled population by examining their special needs and capabilities. For example, decreased travel for a headstick user can be achieved by the use of a center-weighted alphanumeric layout on a miniature keyboard. In contrast, a solution 
Journal of Rehabilitation Research and Development Vol. 29 No. 4 Fall 1992

well suited to one capable of typing at speeds greater than 20 words per minute (smooth and rapid alternation of digits) is irrelevant to the Morse code user who activates two single switches to achieve a rate of eight words per minute.

The following case study vignettes, drawn from our own clinical files, illustrate the application of human factors research to the selection of viable ergonomic data entry device alternatives. The three selected cases are, in many ways, representative of our clinical population. These clients are active adults whose employment potential has been profoundly impaired by the severity of their physical disability. The primary objective for referral pertained to the prescription of microprocessor-based adapted access devices that would increase their vocational options. Their current physical difficulties are related to the original pathological process as well as to postural deformities that have developed gradually. As with most of our clients, they had completed all active in-patient rehabilitative care, and their physical condition was considered to be at a relatively stable level.

\section{Case MM}

MM is a 34-year-old female who has a 2-year history of systemic lupus erythametosus and scleroderma. The disease process, apparently exacerbated by her full-time work as a data processor using a standard electric typewriter keyboard, has led to the development of severe arthritic deformities that include ulnar deviation and boutonniere contractures.

She was referred for adaptive device assessment prior to her admission into a vocational retraining program that will provide instruction in the use of graphics programs on the Macintosh computer. Her primary need concerned the ability to control cursor position. Alphanumeric input, although necessary, was less critical. Our objective was to provide this client with full, direct control of the Macintosh environment without causing further damage to the inflamed joints of the hand.

Consideration of all three of the human factors issues presented above was necessary in this case. Device positioning to alleviate the continued ulnar drift of the metacarpophalangeal (MCP) and proximal interphalangeal (PIP) joints was of paramount importance. Replacement of the Macintosh keyboard was essential to relieve the substantial digit travel and digit loading involved in normal key activation $(39,42,47)$. Finally, an alternative to the Macintosh mouse was required to circumvent the need to grasp and move this device.

The ergonomic solution of choice appeared to be an alternative input control device, the trackball. The trackball serves the same purpose as the more commonly used mouse. The latter device is a palm-sized unit that is propelled over any hard, flat surface. As the mouse is moved, a small ball mounted on its lower surface is rotated; these movements are translated into motions of the screen cursor of proportional magnitude and direction. The trackball differs from the mouse in that the ball is located on its top surface. The ball is rotated directly by very small movements of the index finger (MCP adduction and abduction and PIP flexion and extension). The other digits may assist the index finger or they may remain stationary; the thumb rests on and controls the activation switch.

The characteristics of the trackball seem to be ideally suited to the alleviation of this client's access problems. The small movements required to rotate the ball are much less taxing than the continuous isometric contractions required to clasp and activate the mouse. Moreover, because its operation is not constrained to flat surfaces, the trackball can be positioned at a variety of different angles in accordance with standards developed in device positioning studies $(15,41,42)$. By laterally tilting the trackball to a 45-degree angle and by placing it about $10 \mathrm{~cm}$ below standard table height, the client's forearm is fully supported in a position between extreme pronation and neutral; thus, her hand avoids postures that would foster the development of further ulnar deformities (39). Finally, the trackball, used in conjunction with a keyboard replica program, enables this client to avoid using the standard keyboard. Although this technique is not a particularly efficient method of typing, it is acceptable in the present case because of the relative importance of graphics versus alphanumeric input. An additional feature of the trackball, not required for this client, relates to the separation of cursor movement and command execution. Users with tremor or with fine-motor coordination difficulties are unable to maintain the cursor in its designated location while manipulating the activation switch of the mouse.

In summary, the small cursor displacements, modest activation force, and opportunity for posi- 
tioning make the trackball an excellent method for controlling the Macintosh environment.

\section{Case GT}

GT is a 37-year-old female suffering from dystonia musculorum deformans, a genetic disorder that has resulted in dystonic and spastic irregularities in all of her trunk and $\operatorname{limb}$ movements. Repetitive distorted positioning has left GT with residual orthopedic deformities. She ambulates, with precarious gait, with a cane for short distances but uses an electric wheelchair for outings. GT is independent in personal daily activities and has moderately dysarthric speech. Her right hand is nonfunctional due to severe spasticity and contractures. Using shoulder girdle movements to guide the left $\operatorname{limb}$, she clutches a pen to write or points the third digit to type.

GT's vocational objective was to be employed in a middle management position which would make use of her interpersonal skills, initiative, and organizational abilities. Competitive employment in this capacity would necessitate a thorough knowledge of and hands-on experience with the latest wordprocessing, database, and spread-sheet software; the need to copy-type quickly would be less important. To date, by making use of externally funded adapted transport and note-taking services, GT had coped well in the theoretical courses required by the university level administration program in which she is enrolled.

She was referred for adapted access assessment as soon as her inability to operate the standard PC keyboard became apparent. The decision to use simple modifications to adapt a standard PC/XTcompatible computer was based on a number of factors related to her physical difficulties and her targeted job market. Her limited financial resources and the suitability of this computer to most business settings led us to consider ways in which it could be adapted to accommodate managerial tasks. Direct access via multiple digit access was not feasible because the combination of severe postural deformities and dystonia essentially limited voluntary function to relatively gross movements of the left upper extremity (2). Indirect access via encoding or scanning was dismissed due to a general bradykinesia that would have rendered either of these methods much slower than the single-digit access that she currently uses to operate a typewriter (2).

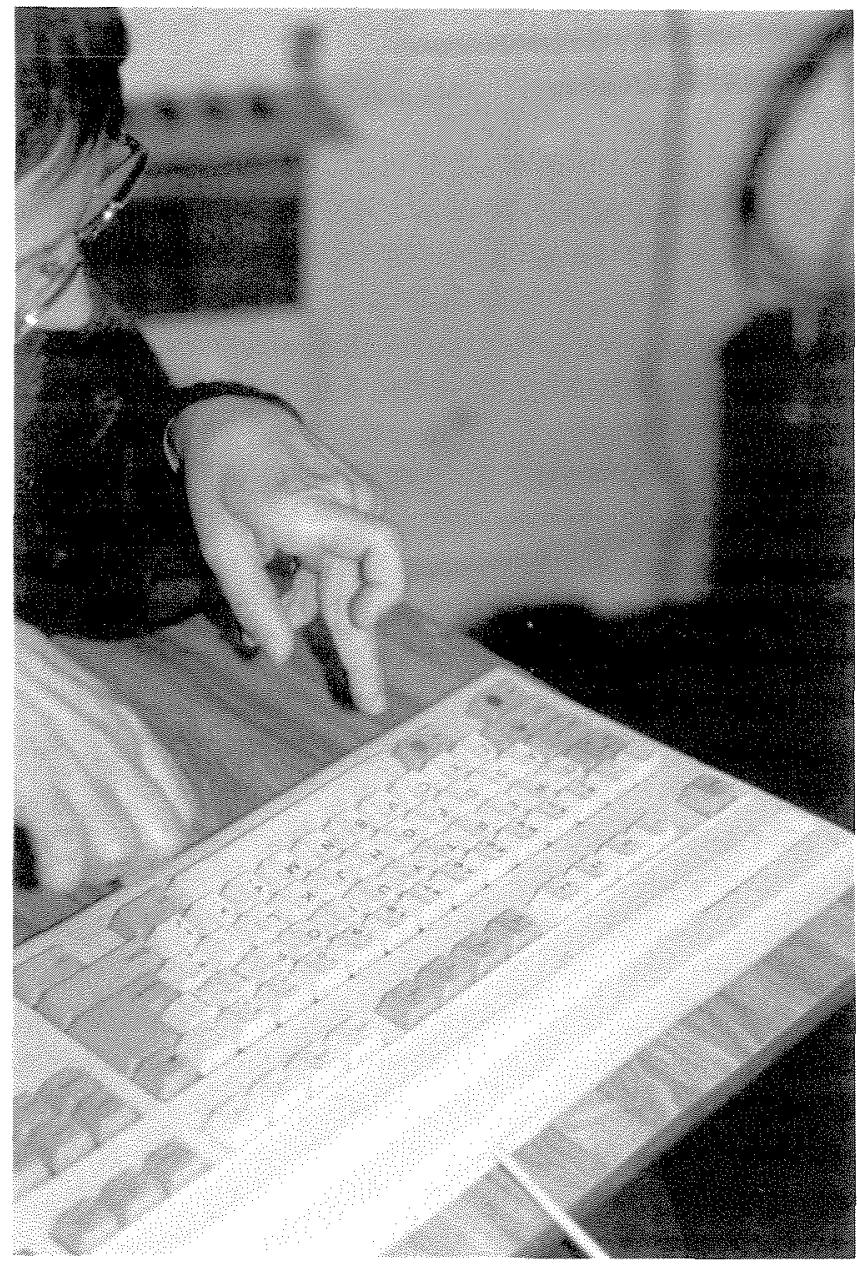

Figure 1.

Client GT using a single-digit typing method to access an $\mathrm{XT} / \mathrm{PC}$-compatible computer.

Our efforts in this case were first directed at providing better postural support $(15,40,42)$. The adjustments available with standard workstation furniture were insufficient for this client due to the severity of scoliotic involvement and her extremely short stature $(39,42,43)$. Within the laboratory environment, proper positioning was achieved with the aid of a six-degree-of-freedom, custom-made adjustable chair. The lateral and coronal plane adjustments provided excellent compensation for trunk asymmetry, whereas movement in the vertical plane permitted appropriate support for her feet and arms. A lap tray provided additional trunk support as well as a surface for placement of the keyboard. Customized foam inserts would enable GT to achieve comparable positioning in the work setting. 
Journal of Rehabilitation Research and Development Vol. 29 No. 4 Fall 1992

We next sought ways to improve her efficiency as a single-digit typist. GT's major access difficulties included the inability to release keys promptly after activation and to strike two or more keys at the same time. A RAM-resident program (1-Finger*, Trace Center, Madison, WI) provided an inexpensive solution to both these problems. This program, compatible with most major software applications, disengaged the key repeat function and allowed simultaneous keystrokes to be replaced by sequential input (Figure 1). GT's efficiency was further improved with the minimization of horizontal digit travel $(1,34)$. To permit GT to continue to use a regular QWERTY keyboard (for reasons of compatibility with standard office equipment), macros were developed to store commonly used phrases. This solution avoided the use of a miniature keyboard (36).

In summary, proper positioning and software innovations have made GT a more efficient singledigit typist. She now uses a PC/XT computer at speeds exceeding 11 words per minute to carry out course assignments and at her volunteer office job.

\section{Case $\mathrm{MC}$}

$\mathrm{MC}$ is a 20 -year-old male with a 3 -year history of multiple sclerosis. Cerebellar involvement resulting in severe visual nystagmus, trunk ataxia, dysmetria, and intention tremor have caused considerable functional limitations for this client. External loading with weights, the provision of limb and trunk support, and emphasis on isometric rather than isotonic actions has greatly enhanced MC's ability to function although he continues to experience substantial difficulties with tasks involving fine motor skill and coordination. He is unable to eat or write independently but can walk with the aid of a wheeled walker provided that he wears a weighted vest.

MC was referred for adapted access assessment to determine whether he would be able to resume the college program in business administration that his illness had forced him to discontinue. His experience with word-processing and database usage as well as his keen interest in and aptitude for programming made him an excellent candidate for computer training. The unsuitability of standard keyboard

*Access DOS, a more recent version of this program, is available from IBM, Inc, at no cost. usage, even when modified by a keyguard, was immediately apparent (2). The severity of MC's intention tremor presented a considerable challenge to the attainment of efficient and productive data entry. MC was unable to cope with the extensive digit travel needed for this input method $(9,10,31)$.

Our objective was to capitalize on MC's ability to execute isometric and quasi-isometric actions accurately provided that the proximal segments were adequately stabilized. Direct access via one of the alternative keyboards that minimize digit displacement seemed, at first, to be a viable option, and MC's ability to type with both the Mini-keyboard (TASH Inc., Ontario, Canada) and the Octima chord keyboard (TASH Inc.) was assessed $(26,27,28)$. Unfortunately, MC was unable to use either keyboard in a functional manner. The reduction in horizontal plane displacement of the Minikeyboard was insufficient to accommodate the severity of his intention tremor, and the vertical plane displacement of the Octima keyboard as well as the coordinated digit movements required to activate it were beyond his capabilities $(26,28)$.

Therefore, Morse code, an indirect access method implemented via the activation of single switches, was considered. Based on switch activation data available in the human factors literature $(7,17,32)$, MC was prescribed with two Minicup switches (TASH, Inc.) having characteristics including size, shape, and activation force and displacement suited to his abilities and activation mode (19). As shown in Figure 2, MC supported each switch between the second and third digit; the switches were activated by slight isometric contractions of opponens pollicis. Note that care had to be taken to select a switch whose activation force was greater than the mass of $\mathrm{MC}$ 's thumb and the force generated by its tremor, yet small enough to prevent fatigue after prolonged usage. The size and shape of the switch were constrained by its placement in the hand. Finally, it was necessary that activation displacement be small enough to prevent the onset of intention tremor. This access method has proven to be highly successful. MC was able to master the Morse code within 2 weeks and has achieved speeds in excess of 10 words per minute. He now uses a variety of word-processing and database programs on a $\mathrm{PC}$-compatible computer via switches that are connected to the PC A.I.D. (Tash Inc.). An added advantage of this access method is that $\mathrm{MC}$ does not 


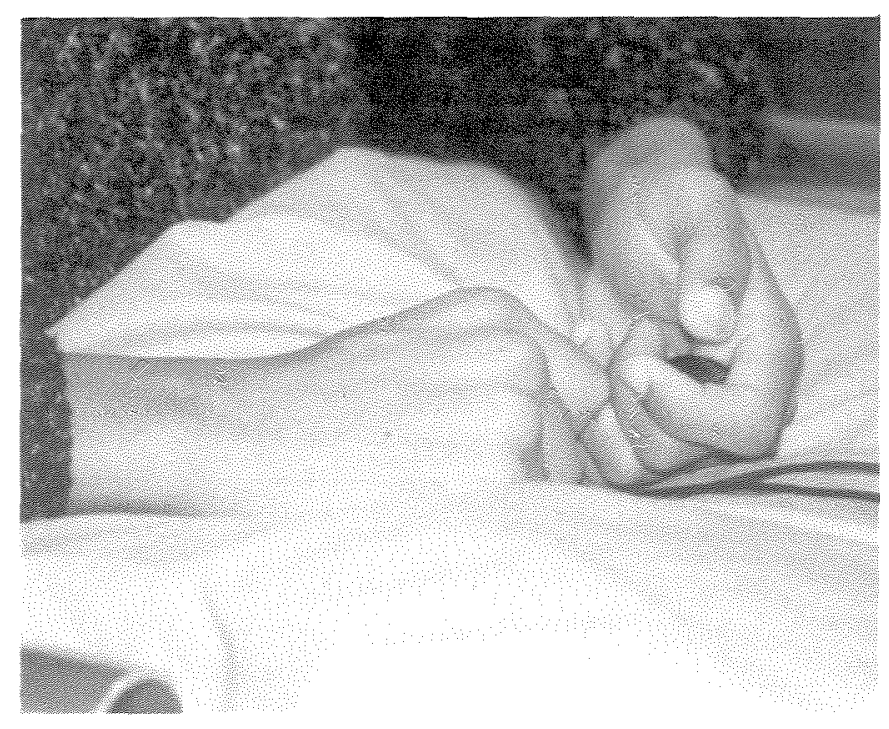

Figure 2.

A close-up view of Client MC using two Minicup switches to operate Morse code.

have to look at the keyboard while typing and is therefore able to continuously support his head, trunk, and arms, thereby minimizing the onset of intention tremor. To augment $\mathrm{MC}$ 's rate of data entry, word prediction and abbreviation-expansion techniques (macros) were recommended $(4,36,48)$. MC's visual limitations make the scanning required for word prediction difficult, but word-processing macros have been quite useful to store commonly used addresses and telephone numbers. In summary, MC has been able to study and work at home, at a summer job, and, most recently, at college with the aid of two adapted switches and a computerized implementation of Morse code.

\section{DISCUSSION}

Microcomputer technology offers numerous opportunities for physically disabled individuals to live with greater autonomy. Within the past decade, integration of the disabled into work and school settings has been facilitated by the provision of adapted data entry devices. As illustrated in the above clinical examples, the adapted access prescription process can be enhanced with the aid of results from human factors research. This information provides therapists with a working framework and substantiates decisions made for device prescription and customization by promoting an awareness of the relative characteristics of the input devices. This approach can generate new ideas for therapists starting out in this field and can encourage the development of ergonomically sound access options by demonstrating the effectiveness of methods not currently used in rehabilitation. Moreover, experimental evidence drawn from the human factors literature lends credibility to financial requests to fund new and replace outdated equipment.

The three cases histories presented in this paper illustrate the contribution that human factors data can make to the adapted access prescription and customization process. Specific human factors hypotheses need to be tested on the disabled population in order to answer questions such as how or if the input speed influences the potential benefits to be gained by the use of alternate hands. However, many of the human factor issues and the hardware and software solutions reviewed here are applicable to individuals with disabilities. Some of the most urgent research needs at this time include the development and testing of: 1) quantitative evaluation programs that enable the measurement of user speed, accuracy, and endurance; 2) novel input methods that make use of the ability of the user to exert a finer level of control within his or her limited range of mobility and strength; and, 3) comprehensive, portable, and affordable text-to-speech synthesizers. It is hoped that an increasing awareness on the part of clinicians of the applicability of human factors research will, in turn, act to mobilize researchers to address these issues of specific concern to the disabled population.

\section{ACKNOWLEDGMENTS}

S. August was supported by a graduate student grant from the Medical Research Council of Canada. This work was facilitated by financial support from the Rick Hansen Man in Motion Legacy Fund and the Canadian Paraplegic Society.

\section{REFERENCES}

1. Chubon RA, Hester MR. An enhanced standard computer keyboard system for single-finger and typing-stick typing. J Rehabil Res Dev 1988;25(4):17-24. 
Joumal of Rehabilitation Research and Development Vol. 29 No. 4 Fall 1992

2. Lee $\mathrm{K}$, Thomas $\mathrm{D}$. Control of computer-based technology for people with physical disabilities: an assessment manual. 1st ed. Toronto: Univ. of Toronto Press, 1990.

3. Wright $\mathrm{C}$, Nomura M. From toys to computers: access for the physically disabled child. Rev, ed. San Jose (CA): Christine Wright, 1988.

4. Smith RO, Christiaansen $R$, Borden $B$, Lindberg $D$, Gunderson J, Vanderheiden G. Effectiveness of a writing system using a computerized long-range optical pointer 10-branch abbreviation expansion. J Rehabil Res Dev $1989 ; 26(1): 51-62$.

5. Vanderheiden GC. Service delivery mechanisms in rehabilitation technology. Am J Occup Ther 1987;41:703-10.

6. Weiss PL. Mechanical characteristics of microswitches adapted for the physically disabled. J Biomed Eng $1990 ; 12: 398-402$.

7. August S, Weiss PL. Objective and subjective approaches to the evaluation of adapted input devices. J Biomed Eng $1992 ; 14: 117-25$.

8. Sanders ME, McCormick EJ. Human factors in engineering and design. 6th ed. New York: McGraw-Hill, 1987.

9. Alden DA, Daniels RW, Kanarick AF. Keyboard design and operation: a review of the major issues. Hum Factors 1972;14:275-93.

10. Noyes $J$. The QWERTY keyboard: a review. Int $J$ Man-Mach Stud 1983;18:265-81.

11. Dvorak A. There is a better typewriter keyboard. Natl Bus Educ Q 1943;12:51-8.

12. Cooper WE. Cognitive aspects of skilled typewriting. 1st ed. New York: Springer-Verlag, 1983.

13. Lahy $\mathbf{J M}$. Motion study in typewriting. International Labour Office, Studies and Reports Series J. (Education) no. 3, 1924.

14. Kinkead R. Typing speed, keying rates, and optimal keyboard layout. Proceedings of the Human Factors Society 19th Annual Meeting; 1975; Dallas (TX). : 159-61.

15. Ferguson D, Duncan J. Keyboard design and operating postures. Ergonomics 1974;17:731-44.

16. Technology Update (column). Can I Occup Ther $1990 ; 57: 47-8$.

17. Yamada H. A historical study of typewriters and typing methods: from the position of planning Japanese parallels. J Inf Process 1980;2:175-202.

18. Norman DA, Fisher D. Why alphabetic keyboards are not easy to use: keyboard layout doesn't much matter. Hum Factors 1982;24:509-19.

19. West LJ. Vision and kinesthesis in the acquisition of typewriting skill. J Appl Psychol 1967;51:161-6.

20. Gentner DR. Keystroke timing in transcription typing. In: Cooper WE, editor. Cognitive aspects of skilled typewriting. New York: Springer-Verlag, 1983: 95-117.

21. Larochelle S. A comparison of skilled and novice performance in discontinuous typing. 1st ed. In: Cooper WE, editor. Cognitive aspects of skilled typewriting. New York: Springer-Verlag, 1983: 67-91.

22. Fox JG, Standsfield RG. Digram keying times for typists. Ergonomics 1974;17:731-44.

23. Ostry DJ. Determinants of interkey times in typing. In: Cooper WE, editor. Cognitive aspects of skilled typewrit- ing. New York: Springer-Verlag, 1983: 225-43.

24. Deininger RL. Human factors engineering studies of the design and use of push button telephone sets. Bell Systems Tech J 1960;39:995-1012.

25. Gopher D, Raji D. Typing with a two handed chord keyboard: will the QWERTY become obsolete? IEEE Trans Syst Man Cybern 1988;18:601-9.

26. Ratz HC, Ritchie DK. Operator performance on a chord keyboard. J Appl Psychol 1961;45:303-8.

27. Conrad R, Longman DJA. Standard typewriter versus chord keyboard-an experimental comparison. Ergonomics 1965;8:77-88.

28. Kirschenbaum A, Freidman Z, Melnik A. Performance of disabled persons on a chordic keyboard. Hum Factors 1986;28:187-94.

29. Minor FJ, Revesman SL. Evaluation of input devices for a data setting task. J Appl Psychol 1962;46:332-6.

30. Albert, A. The effect of graphic input devices on performance in a cursor positioning task. Proceedings of the Human Factors Society 26th Annual Meeting. Santa Monica (CA): Human Factors Society, 1982.

31. Brunner $\mathbf{H}$, Richardson $\mathbb{R}$. Effects of keyboard design and typing skill on user keyboard preferences and throughput performance. Proceedings of the 1984 International Conference on Occupational Ergonomics. 1984: 267-71.

32. Loeb KM. Membrane keyboards and human performance. Bell System Tech J 1983;62:1773-48.

33. Sternberg $S$, Monsell S, Knoll RL, Wright CE. The latency and duration of rapid movements sequences: comparisons of speech and typewriting. In: Stelmach GE, editor. Information processing in motor control and learning. New York: Academic Press, 1978: 117-52.

34. Kroemer KH. Human engineering the keyboard. Hum Factors 1972;14:51-63.

35. Hooleon Corp. Custom keyboard products. Cornville (AZ): Hooleon, 1990.

36. Demasco $\mathrm{P}$, Lillard $\mathrm{M}$, McCoy $\mathrm{K}$. Word compansion: allowing dynamic word abbreviations. Proceedings of the 12th Annual RESNA Conference; 1989 June 25-30; New Orleans (LA). Washington (DC): RESNA Press, 1989: 282-3.

37. Vanderheiden GC. Practical application of microcomputers to aid the handicapped. Rehabil Lit 1981;44:66-70.

38. Nakaseko E, Grandjean E, Hunting W, Gierer R. Studies on ergonomically designed alphanumeric keyboards. Hum Factors 1985;27:175-87.

39. Green RA, Briggs CA. Effect of overuse injury and the importance of training on the use of adjustable workstations by keyboard operators. J Occup Med 1989;31:557-62.

40. Lundervold A. Electromyographic investigations during typewriting. Ergonomics 1958;1:226-33.

41. Komoike $\mathrm{Y}$, Horiguchi S. Fatigue assessment on key punch operators, typists and others. Ergonomics 1971;14:101-9.

42. Grandjean E. Fitting the task to the man: a textbook of occupational ergonomics. 4th ed. London: Taylor \& Francis, 1988.

43. Miller W, Suther TW. Display station and anthro- 
pometrics: preferred height and angle settings of CRT and keyboard. Hum Factors 1983;25:401-8.

44. Scales EM, Chapanis A. The effect on performance of tilting the toll-operator's keyset. J Appl Psychol 1954;38:452-6.

45. Fess EE, Gettle KS, Strickland JW. Hand splinting principles and methods. 1st ed. St. Louis (MO): CV Mosby, 1981.

46. Creamer LR, Trumbo D.A. Multifinger tapping performance as a function of the direction of tapping move- ments. J Appl Psychol 1960;44:376-80.

47. Erdelyi A, Sihvonen T, Helin P, Hanninen O. Shoulder strain in keyboard workers and its alleviation by arm supports. Int Arch Occup Environ Health 1988;60:119-24.

48. McCoy K, Demasco P, Gong Y, Pennington C, Rowe C. Toward a communication device which generates sentences. Proceedings of the 12th Annual RESNA Conference; 1989 June 25-30; New Orleans (LA). Washington (DC): RESNA Press. 1989: 149-50. 